\title{
Dr. Xue Qin Yu: social determinants in health should be an important subject in medical education
}

Submitted Jul 19, 2018. Accepted for publication Jul 25, 2018.

doi: $10.21037 / \mathrm{atm} .2018 .07 .31$

View this article at: http://dx.doi.org/10.21037/atm.2018.07.31

\section{Editor's note}

Dr. Xue Qin Yu is an honourable Editorial Board Member of Annals of Cancer Epidemiology (Ann Cancer Epidemiol; ACE; ISSN: 2616-4213) and has recently participated in a focused issue under the theme of "Global cancer burden". Fascinated about the area of cancer epidemiology, Dr. $\mathrm{Yu}$ and his research team has spent years in conducting various population-based analyses on the major cancers both in Australia and China. This time, we are honoured to interview Dr. Yu to share with our readers some of his most important research findings and another inspiring cause that he has recently initiated.

\section{Expert's introduction}

Xue Qin Yu, PhD, MPH, Senior Research Fellow, Cancer Research Division, Cancer Council NSW, PO Box 572, Kings Cross, NSW 1340, Australia.

Dr. Xue Qin Yu is a Senior Research Fellow in the Cancer Research Division at Cancer Council NSW, and an Adjunct Senior Lecturer at the University of Sydney School of Medicine, Australia (Figure 1). He was the recipient of a prestigious NHMRC Early Career Fellowship and a Prostate Cancer Foundation of Australia Young Investigator Award. He is an invited reviewer for national and international funding bodies, and regularly provides reviews for 33 national and international scientific journals since 2008.

Xue Qin's primary research focus is cancer epidemiology, statistical modelling and health services research. His current area of interest is cancer disparities and the social determinants of health and health outcomes research, with a focus on the development and application of statistical tools to evaluate the cancer health care system and explore the possible reasons for cancer disparities.

Xue Qin has a strong track record in peer-reviewed publications, having co-authored $60+$ peer-reviewed articles. During the last 5 years, he co-authored 37

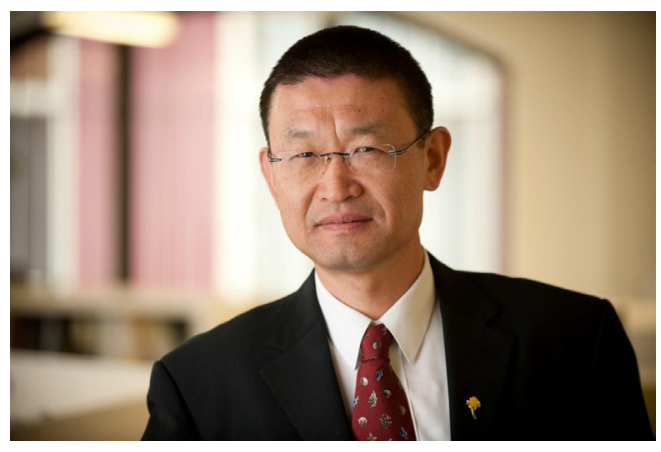

Figure 1 Dr. Xue Qin Yu.

articles, with 20 of which as first/last author. His research has been published in many prestigious journals, including CA: A Cancer Fournal for Clinicians, Lancet Global Health, and Lancet Public Health.

\section{Interview}

\section{ATM: When did you start the research on cancer epidemiology and what attracted you?}

Dr. Yu: I started the research on cancer epidemiology from 2000. My supervisor at that time was Professor Bruce K Armstrong (a fellow of the Australian Academy of Science since 2000) who has led me to this area. He is arguably the most outstanding public health scientist in the history of Australian epidemiology.

\section{ATM: Could you briefly introduce to us your research team?}

Dr. Yu: I have supervised three higher degree research students (all with the University of Sydney, Australia), and also trained three international visiting fellows at different time periods, who all worked on different projects. The three higher degree research students are:

Julia Stanbury who worked on the projects examining 
survival differences among various population groups for many cancer types, and her thesis was completed in 2016.

Qingwei Luo has mainly studied on the unknown stage of prostate cancer recorded in the cancer registry, its impact on epidemiological studies using this variable, and how to deal with its impact in such studies, and she will complete her thesis in a couple of months.

Dr. Elvin Cheng has just started his research with me in January this year, and he is studying the possible risk factors for lung cancer amongst never-smokers. In this project, we will use large cohort datasets which are accessible in Australia (the 45 and Up Study) and China (China Kadoorie Biobank). Both cohorts are large, with the Chinese cohort over half a million and the Australian one over 260,000 participants.

\section{ATM: What are the major cancers you study on at the moment?}

Dr. Yu: I am currently studying the four major cancers in Australia, namely colorectal cancer, lung cancer, female breast cancer and prostate cancer. These cancers have a big impact among the population, accounting for about $50 \%$ of the total cancer incidence in Australia.

\section{ATM: What are the challenges in conducting statistical modelling in cancer research?}

Dr. Yu: There are three points worth mentioning. The first is regarding the sample size. When dealing with small sample size, it is challenging to select an appropriate statistical technique to handle its large variation. There are many studies that have limited number of participants, and it is very important to choose an appropriate statistical method and interpret the results accordingly. The second is that we need to use both relative and absolute measures. Many research projects apply only relative measures, yet absolute measures are also very important from the public health point of view, but are not used as often. The third is to be aware of the relationship between statistical significance and clinical importance. Sometimes one may find a risk factor with high statistical significance, however, it may affect only a small number of patients, so the clinical importance of this risk factor may not be that high.

\section{ATM: What does health services research involve?}

Dr. Yu: It is mainly about the investigation on how people use medical services. Health services may be overused by certain groups but are underutilised among others. This will consequently affect the disease outcomes among different population groups. This will also include the cost-benefit analysis among the groups with different patterns of using health services.

\section{ATM: Are there any research projects underway that you would like to share with us? What can we expect?}

Dr. Yu: One of the main projects that I am currently involved in is about the investigation on the reasons why certain population groups have lower cancer survival than the others. The prognostic factors can affect a cancer patient at different stages, from pre-diagnosis, diagnosis, treatment to post-treatment. We have used the data from the 45 and Up Study, which is the largest cohort in Australia. The baseline information was linked with several health-related datasets to cover the whole care pathways from how patients got diagnosed, how they were treated, to how long they had survived. Thus, we can figure out why certain people have poorer survival than others, then, we can further develop appropriate interventions to reduce the survival disparities and to improve the outcomes for the whole population.

\section{ATM: What would you like to do apart from scientific research?}

Dr. Yu: I am the president of a newly established association, China Anti-Cancer Association Australia (CACA Australia). What we would like to do is to provide a platform for the collaboration of the professionals in cancer research and clinicians in Australia and China. We would like to do some good things for the Chinese immigrants in Australia, specifically, to provide some services to those who are affected by cancers.

\section{ATM: Are there enough medical oncologists and other cancer specialists to care for cancer patients in Australia?}

Dr. Yu: The emerging evidence has shown that we will need more medical oncologists because of the increase in cancer survivors. My own research suggests that the number 
of cancer survivors will increase substantially in the future in Australia. Therefore, we will need more cancer specialists including oncologists to provide different types of care required by cancer survivors as they move through the disease trajectory. However, another key issue is how to allocate limited medical resources in a more balanced and cost-effective way, so that populations from rural and remote areas or those of socioeconomically disadvantaged can have adequate access to the services they need as well.

\section{ATM: Can you briefly introduce to us the geographic variation in prostate cancer survival in New Soutb Wales?}

Dr. Yu: In a study of geographic variation in prostate cancer survival in New South Wales, we have found that patients from rural and remote areas have lower cancer survival than those patients in major cities. Taking account of the timeliness of diagnosis into the analysis does not change the finding, indicating that access to specialists/treatment may be a significant reason for this variation.

\section{ATM: Are most Australian women aware of the bealth care needs if diagnosed with breast cancer?}

Dr. Yu: Some population groups, for example the new arrival migrants or those from non-English speaking backgrounds, may not know the health system in Australia very well. This may likely affect the timeliness of their diagnosis in the first place, and then after diagnosis it is very difficult for them to navigate the complex health system to get appropriate treatment. In addition, as most patients normally can survive for a long period of time, it is important to help those in need to gain access to the different healthcare services available after initial treatment.

\section{ATM: How do you think of the future cancer burden in China? Will there be any differences in terms of bealth care services required in comparison to Australia?}

Dr. Yu: The cancer profiles are different in these two countries. In Australia, the top five cancers are cancers of the colorectum, lung, breast, prostate and melanoma of the skin. Among them, only lung cancer is highly fatal. While in China, the top five major cancers include cancers of the lung, stomach, esophagus, liver and colorectum, most of which are fatal cancers (except for colorectal cancer). Thus, the health care services as required are quite different between the two countries. Regarding the cancer control in China, I believe primary prevention is much more important (and more cost-effective) than treatment, as the treatments for these major cancers (except for colorectal cancer, or unless detected at an early stage) are not very effective.

ATM: What would be your suggestions for policy makers in Australia to plan for future cancer services?

Dr. Yu: There are many things the government can do for cancer control in Australia. According to my research, I found that addressing the social demographic disparity can have a great impact on cancer incidence and cancer survival, thus targeted interventions may be a costeffective way to reduce cancer burden for the whole population. In my opinion, the government should proportionally allocate more health-care resources to the disadvantaged populations to improve cancer care, which will potentially reduce the survival disparities between population groups.

ATM: What would be your advice to the young medical professionals in Australia?

Dr. Yu: Currently, the social determinants of health are not emphasised enough in the medial education in Australia. Most students do not know much about the social disparity in health. People may assume that with the universal health cover, everyone can achieve good health outcome. However, that is not the case. From our studies, certain population groups had poorer health outcomes for a range of different cancers. Despite overall increases in cancer survival over time in Australia, socioeconomically disadvantaged groups had much slower increase, thus disparities had actually become worse over time for certain cancers. I think we need to introduce this subject to the medical schools' curriculum so that the medical students will have this kind of training to equip them with an understanding of social determinants of health outcomes. This would be critical to developing a generation of medical professionals with the knowledge and skills necessary to address these disparities.

\section{Acknowledgements}

We would like to express our sincerest gratitude to Dr. Xue Qin Yu for sharing his insights and opinions with us. 


\section{Footnote}

Conflicts of Interest: The views expressed in this interview are those of Dr. Xue Qin Yu based on his past research and

Cite this article as: Kong V. Dr. Xue Qin Yu: social determinants in health should be an important subject in medical education. Ann Transl Med 2018;6(15):311. doi: 10.21037/atm.2018.07.31 do not necessarily reflect the views of his employer (Cancer Council NSW, Australia).

(Science Editor: Vivian Kong, ATM, editor@atmjournal.org) 